\title{
Le monde urbain et ses rencontres : entre délocalisation et réappropriations
}

\author{
« La modernité, c'est le transitoire, le fugitif, le contingent ${ }^{1}$... »
}

Qu'advient-il de nos rencontres et quelles formes prennent-elles dans le contexte d'une hypermodernité — c'est-à-dire une modernité radicalisée, selon l'idée d'Anthony Giddens $^{2}$ - dont les traits significatifs sont un phénomène de délocalisation généralisé et une « urbanisation des mœurs ${ }^{3} »$ ?

Ce texte voudrait contribuer à une interrogation sur les nouvelles façons de vivre la ville et les relations sociales, étant donné l'intégration des technologies de l'information et de la communication (TIC) dans la vie quotidienne. Il s'agit, comme l'a écrit Alain Mons, de «restituer la rationalité et l'insaisissable qui constituent les deux développements contradictoires des sociétés modernes ${ }^{4}$ ou encore de repérer «les objets de création» derrière « les objets de déterritorialisation $»^{5}$. Il sera ainsi question du sens des pratiques dans le monde d'aujourd'hui, où s'entrecroisent culture numérique et culture urbaine où se combinent dématérialisation et réappropriation de l'espace et des contacts. Deux activités récentes seront décrites et proposées en exemple. Je parlerai d'abord du speed dating, mais il sera surtout question par la suite du bookcrossing.

Il me faut préciser que les réflexions qui vont suivre sont issues d'une enquête en cours. Celle-ci est, pour le moment, majoritairement basée sur une étude documentaire (observation des sites Internet concernés) et sur une observation participante (rencontre des utilisateurs, participation aux activités du bookcrossing). Elle ne prétend pas à une représentativité statistique mais a plutôt pour intention de relater et comprendre des pratiques dont on pourrait se demander si elles n'expriment pas quelque chose de symptomatique pour notre époque. Le bookcrossing n'est pas, en effet, la seule activité de la sorte. Il n'y a pas que des livres qui circulent entre Internet, les individus et les lieux publics. Des billets de banque, des appareils photographiques et autres « trésors ${ }^{6}$ sont aussi échangés. Ainsi, bien que les contenus de ces activités varient ; formes et moyens se répètent (circulation d'un objet-relais, déplacement dans un espace public physique, gestion des contacts et traçage des objets par le biais d'Internet).

\footnotetext{
${ }^{1}$ Charles Baudelaire, Petits poèmes en prose (le spleen de Paris), Paris, Pocket, 1995, p. 170.

2 Voir Anthony Giddens, Les conséquences de la modernité, trad. de l'anglais par Olivier Meyer, Paris, L'Harmattan, 2002, p. 155.

${ }^{3}$ Patrick Baudry et Thierry Paquot (textes réunis par), L'urbain et ses imaginaires, Pessac, MSH d'Aquitaine, 2003, p. 9.

${ }^{4}$ Ibid, p. 10.

${ }^{5}$ Ibid., p. 11.

${ }^{6}$ Voir les sites Web suivants : Phototag permet le traçage d'appareils photographiques jetables que les membres du réseau ont fait circulé. Les photos prises sont ensuite mises en ligne (http://www.phototag.org/, consulté le 12 avril 2008). Eurobilltracker a pour objectif le traçage des billets en euros à travers le monde (http://fr.eurobilltracker.com/, consulté le 12 avril 2008). Enfin Geocaching propose une chasse au trésor, à l'aide du GPS, avec des " géocacheurs » et des « géotrouveurs » d'objets (http://www.geocaching-france.com/, version française, consulté le 12 avril 2008). Enfin le site http://www.confluence.org a pour projet la visite de chaque point géographique d'intersection entre longitude et latitude sur la planète. Les photos de ces « carrefours » imaginaires sont ensuite mis en ligne et répertoriées sur le site (consulté le 13 avril 2008).
} 


\section{Le speed dating : des rencontres chronométrées}

Régulièrement, des rencontres sont orchestrées soit sur des sites Internet soit à la télévision, via des émissions faiseuses de «lien social». Ces dernières se donnent à voir comme les médiateurs de ce qui soi-disant ne se ferait plus tout seul (alors que les entremetteurs ne datent pas d'hier, ils ont seulement changé de forme). Cependant l'instrumentalisation de ces événements (service payant, publicité, systématicité de l'organisation) pose question. Avec le développement d'Internet, nous ne disposons pas seulement de nouveaux moyens technologiques, nous n'avons pas uniquement accès à une communication multimédiatique, c'est aussi tout une idéologie qui nous est transmise et qui dépasse les frontières propres de son usage. Idéologie du branchement simultané, du choix multiple instantané et plus que jamais, de la disponibilité.

Ainsi de nouvelles formes d'organisation de la rencontre se développent à l'image de la forme Web. Les fameux «speed dating » (littéralement : rendez-vous express), activité qui existe maintenant depuis plusieurs années, ont lieu certes dans la «vraie» vie mais se préparent d'abord sur Internet ${ }^{l}$. Le principe : des rencontres entre célibataires sont organisées dans un bar ou un café à l'ambiance soignée. Des candidats font connaissance pendant un temps minuté (le plus souvent moins de dix minutes) et enchaînent ainsi les rendez-vous dans une même soirée. A la fin, chacun remplit une fiche à remettre aux organisateurs et sur laquelle il a émis ses choix. L'inscription aux sessions est payante et s'enregistre sur Internet.

Il existe aussi des speed dating dans le recrutement au travail. On y trouve le même type d'organisation: des candidats à l'emploi enchaînent des entretiens éclair avec des recruteurs : "Véritable opération de séduction : entreprises, candidats, recrutez-vous, vous avez sept minutes pour vous convaincre $»^{2}$ représente le genre de slogan que l'on peut lire sur les sites Web. Si l'on imagine bien qu'il ne s'agit que d'un premier contact, dans l'un comme dans l'autre cas, la démarche relève de la croyance en la révélation professionnelle ou amoureuse. Ce qui interpelle ici, ce n'est pas tant le lien au mythe Internet de l'ouverture sans limite que le parallélisme constaté entre les conceptions de la recherche d'emploi et de l'âme sœur. Ainsi recruterait-on son partenaire comme on recrute en entreprise et inversement : l'entreprise qui "s'humanise » va à la rencontre des candidats en espérant « tomber » sur la bonne personne, comme on tombe amoureux. De près ou de loin, les speed dating visent ainsi l'efficacité et fonctionnent comme des usines à coup de foudre (non garanti), en pratiquant le taylorisme de la rencontre.

Ces stratégies relationnelles liées à Internet ont pour effet un premier paradoxe : en même temps qu'elles facilitent la prise de contact, les TIC en tant que système expert, produisent un monde "d'accès distants», un monde de relations partiellement dématérialisées et mixées d'images multimédiatiques. Ainsi nous n'incorporons plus simplement un environnement, des contraintes, des idéologies mais aussi des images et en particulier des «images de soi » à travers les outils de la vie en réseau : e-mail, pseudos, profils et comptes personnels enregistrés sur les différents sites visités. Curieusement, ces possibilités de connexions ou de branchements multiples ont pour conséquence une sorte d'abstraction. La multiplication des images et des soi virtuels produit un individu contemporain qui se distancie de lui-même. Le phénomène des émissions de « téléréalité » où des personnes sont filmées en continu montre d'ailleurs comment le fait d'être exposé ne montre finalement rien; rien d'autre que des images de soi qui ne pénètrent jamais l'intimité des acteurs pourtant scrutée par les caméras et autres Webcams. Le phénomène des «blogs »

\footnotetext{
${ }^{1}$ Voir par exemple les sites http://www.turbo-dating.com/ et http://www.selectperfect.com/ (consultés le 13 avril 2008).

${ }^{2}$ Voir par exemple le site http://www.speed-dating-emploi-dijon.com/ (consulté le 13 avril 2008).
} 
n'échappe pas à cette logique. Ces minis sites Web qui se donnent souvent à voir comme des (pseudo-)journaux intimes ne sont jamais qu'une exposition égotiste de soi partielle et idéalisée, sortes de masques au bal costumé du virtuel. C'est cela la force et le paradoxe de l'imagerie (multi)médiatique: l'immédiateté en même temps que l'infinie distance, l'instantané en même temps que l'insignifiance et l'écart silencieux et énigmatique d'une intimité finalement insaisissable puisqu'elle échappe à la visualisation. Ce que Patrick Baudry a très justement écrit au sujet de la pornographie pourrait être redit, excepté qu'ici le candidat au speed dating ou le blogueur sont les créateurs de leur propre image : "Se chosifier soimême en entrant dans l'apparence des choses, dans leur incongruité et leur puissance, leur capacité de ne rien signifier, et tout à la fois, restituer à la platitude de l'image le hors champs du secret que partagent ceux qui se donnent à la mise en scène du simulacre. $\rangle^{1}$ Ainsi, l'intime se retranchant indéfiniment, les corps deviennent des images. Les images reflètent des corps. Et l'on assiste dans les rencontres à un croisement d'images de soi qui font écran dans le corps à corps concret avec autrui. Le cas des rencontres sur Internet est à ce titre exemplaire. Dans cette production de la surface, on serait ainsi tenté de penser que plus on montre, moins on voit.

Georg Simmel, au début du XXe siècle, avait déjà souligné cette évolution du psychisme et des relations sociales vers l'abstraction, évolution qu'il attribuait au développement des grandes villes. Avec le concept de délocalisation généralisée (laquelle a lieu à tous les niveaux de la vie sociale), Anthony Giddens confirme cette idée dans son essai sur les conséquences de la modernité ${ }^{2}$. Dans le cas du speed dating, étant donné son mode d'organisation (les candidats par exemple s'engagent à ne pas se communiquer directement leurs adresses non plus à se dire directement s'ils souhaitent se revoir), et le fait même que cela soit organisé, on peut raisonnablement se demander dans quelle mesure la découverte d'autrui est réellement favorisée, même si elle n'est pas impossible, loin s'en faut.

Un second paradoxe naît de l'organisation multimédiatique de ces rencontres : malgré la prise en charge de la mise en contact par les médiations techniques -, la réussite d'une rencontre — et c'est là sa spécificité — n'est jamais garantie. Le paradoxe est d'autant plus significatif que nous vivons dans une société dite « de communication », c'est-à-dire, suivant André Akoun et Paul Ansart, une société qui propose les moyens technologiques d'une «communication sans limite ni de publics ni de distances». Ainsi «l'ubiquité abolit le lointain $»^{3}$. En raison de l'augmentation des contacts possibles par le biais d'Internet, les rapprochements devraient être facilités. Dans l'exemple des speed dating, l'idée de disponibilité était implicite mais omniprésente : en multipliant les entrevues, en mettant à disposition plusieurs candidats «libres », on augmente logiquement — c'est-à-dire statistiquement - les «chances de rencontre». C'est ici qu'il faut noter les deux sens contenus dans le même mot: on multiplie les rencontres pour augmenter les chances de rencontre... L'écart signale la différence entre une vision quantitativiste et objectiviste et une vision qualitativiste et subjectiviste de la rencontre. D'un côté la rencontre comme entrée en contact, dénombrable, observable, répétable; de l'autre la rencontre unique, pour une part incontrôlable et toujours surprenante. La première s'organise - entre autres sur Internet la seconde désorganise et ne prévient jamais lorsqu'elle arrive.

\footnotetext{
${ }^{1}$ Patrick Baudry, La pornographie et ses images, Paris, Armand Colin, « Chemin de traverse », 1997, p. 132.

${ }^{2}$ Voir le passage sur la réflexivité qui est, selon le sociologue anglo-saxon, l'expression psychique de cette délocalisation (Anthony Giddens, op. cit., p. 43).

3 André Akoun et Pierre Ansart, «Présentation», Cahiers Internationaux de Sociologie, vol. CXII («Communication et liens sociaux »), janv-juin 2002, p. 5.
} 
Avec ce court exemple du speed dating, il serait aisé d'en conclure que le monde moderne, monde de la dé-liaison, ne permet plus de se rencontrer... Cependant, les choses ne sont pas si simples. D'abord, il n'est pas sûr que nos rencontres soient moins « authentiques » qu'«avant», quand la communauté arrangeait les unions, forçait à la cohabitation intergénérationnelle dans les familles, prescrivait les fréquentations. Par ailleurs, les rencontres (amoureuses et autres) ne peuvent plus être considérée de la même manière " qu'avant», dans un contexte contemporain marqué par l'urbanisation, l'individualisme et l'introduction en masse des images et des «NTIC » dans l'espace social. Nous ne pouvons plus en effet opérer sans faire avec les images. Ainsi, dans le monde d'aujourd'hui, monde hypermoderne, la rencontre ne peut être ni pensée ni vécue hors de son spectacle. Il n'y a pas d'un côté une ou des images médiatiques pures et irréelles de la rencontre et, de l'autre, des expériences pures de rencontre dénuées d'imagerie. C'est un fait de modernité que la combinaison, l'intrication de l'image et du vécu, aujourd'hui plus que jamais avec l'éclosion $\mathrm{du}$ « virtuel» dans nos foyers. Ainsi pas de rencontre sans image(s) de la rencontre. Enfin, comme l'ont montré plusieurs sociologues ${ }^{1}$, une pensée de la modernité urbaine serait incomplète sans la prise en compte de sa complexité. Or, à côté des effets aliénants, se développent aussi des «pratiques singulières » qui semblent exprimer un désir de réappropriation et la recherche d'un retour à une forme de proximité, malgré et par le numérique. A ce titre, peut-être pourrait-on parler du «bookcrossing " comme l'un de ces objets de création qui permettent d'approcher «l'impalpable de la ville $»^{3}$.

\section{Le bookcrossing : une tentative de réappropriation symbolique ?}

$\mathrm{Au}$ carrefour du local et du mondial, du virtuel et du réel, de l'image et du «corps métropolitain ${ }^{4}$, le bookcrossing est une pratique à la fois culturelle et urbaine assez récente ${ }^{5}$. Il se veut être une alternative aux canaux traditionnels de transmission de la culture livresque (achat dans les librairies, emprunt dans les bibliothèques).

Le bookcrossing se présente comme une association libre sans statuts déposés, avec un site $W e b$ officiel, créé et géré par l'initiateur du mouvement ${ }^{6}$. L'idée est née d'un informaticien américain qui s'inspira d'autres pratiques semblables (essentiellement la circulation d'objets et leur traçage sur Internet $)^{7}$. Pour l'instant, la chaîne d'échange est plutôt fragile puisque, d'après ce qui est dit sur le site, seuls $20 \%$ des livres sont retrouvés, le reste étant perdu (il y a, à ce propos, une rubrique « cimetières des livres » pour signaler les pertes). Le site indique plus de 660000 inscrits dans le monde entier ${ }^{8}$.

Comme son nom l'indique, le bookcrossing met en jeu des livres. En résumé, des lecteurs feignent d'oublier ou « abandonnent » un ouvrage dans un endroit public, en espérant

\footnotetext{
${ }^{1}$ Voir Alain Mons, La métaphore sociale : image, territoire, communication, Paris, PUF, 1992 ; Patrick Baudry, Violences invisibles : corps, monde urbain, singularité, Bègles, Ed. du Passant, 2004.

${ }^{2}$ Alain Mons, op. cit., p. 11.

3 Ibid., p. 66.

${ }^{4}$ Ibid., p. 192.

${ }^{5}$ Voir les articles de presse publiés à ce sujet. Par exemple : « Le cercle des "passeurs de livres" », Le Monde, 26 septembre 2003 ; "Passeurs d'histoires », Libération, 6 novembre 2003 ; "Une chasse aux livres lancée ce samedi », Le Figaro, 19 mai 2005 ; " Modiano dans un lavomatic » Le Nouvel Observateur, 21-27 juillet 2005 ; " Au rendez-vous des livres voyageurs », Libération, 29 novembre 2005 ; "'Lire en fête', mode d'emploi », Le Monde des livres, 13 octobre 2006 ; «Des livres à s'échanger dans les lieux publics », Ouest France, 4 juillet 2007 ; « La folie bookcrossing : 'On est toujours là !' », Le Progrès, 1 er août 2007.

${ }^{6}$ Le site Web officiel est http://www.bookcrossing.com (consulté le 13 avril 2008). Le site miroir en français est : http://bookcrossingfrance.apinc.org/ (consulté le 13 avril 2008). Il existe un autre site mais avec un public plus réduit et un nombre moindre de villes concernées. Il s'agit du Passe livre, site inauguré par un libraire italien Gennano Capuano, installé à Paris (http://www.passe-livre.com, consulté le 13 avril 2008).

${ }^{7}$ Voir les exemples donnés en début d'article.

${ }^{8}$ Chiffres au mois d'avril 2008.
} 
que ce dernier sera récupéré par un autre lecteur qui le remettra à son tour en circulation. Deux cas de figures se présentent : soit le livre est trouvé accidentellement par un passant (cas le plus fréquent) ou par un membre, soit il est trouvé par un «bookcrosser » qui a relevé ses coordonnées sur le site $W e b$.

Bien qu'il y ait un refus de toute institutionnalisation, de toute commercialisation (encore qu'il y aurait des choses à dire à ce sujet ${ }^{1} \ldots$ ), bien que le seul but affiché soit le simple goût de lire et le plaisir de la rencontre plus ou moins fortuite avec un livre, voire avec son lecteur, l'activité du bookcrossing ne serait pas ce qu'elle est sans son site Web officiel. C'est là que les lecteurs s'inscrivent. Ils s'enregistrent sous un pseudo ce qui leur permet de gérer leur collection de livres, chacun possédant une étagère virtuelle sur laquelle il « range » ses ouvrages. Avant d'être déposés, les ouvrages sont préalablement enregistrés sur le site avec un numéro d'identification reporté sur l'ouvrage. Chaque dépôt et chaque trouvaille sont aussi signalés sur le site (date et lieu). Ce système doit permettre de suivre la trace d'un livre en circulation. Lorsqu'il décide de laisser un livre quelque part et pour éviter que ce dernier soit pris pour un objet perdu, le passeur lui adjoint une étiquette ou un marque-page portant un message expliquant au futur receveur la marche à suivre ${ }^{2}$.

Dans l'idéal, quand tout fonctionne bien, le livre est pisté à travers le monde. On doit pouvoir suivre «la vie du livre» comme disent les bookcrossers. Bien que la réalité ne soit pas toujours à la hauteur des espérances des adeptes (livres perdus, communication sommaire et imprécise sur les lieux et dates de dépôt : trouver un livre dans une zone urbaine revient à peu près à trouver "une aiguille dans une botte de foin »), je ferai «comme si » car je m'intéresse ici aux intentionnalités, essentiellement désirantes et imaginaires. Ainsi à quel rêve de sociabilité urbaine nous convie le bookcrossing?

Cette activité qui nous vient des Etats-Unis s'adresse aux internautes sur le mode l'invitation. Elle affiche une ouverture et une forme de nonchalance vis-à-vis de cadres imposés aux échanges. Le mouvement se donne à voir comme une pratique alternative mais pacifique qui aurait pour résultat la création d'une bibliothèque mondiale sans mur, sans autres règles que le plaisir de lire et le respect de la communauté des lecteurs. Le site annonce ainsi que le bookcrossing est «un grand club de lecture traversant temps et espace, ne connaissant aucune limite géographique », un club de lecture gratuit avec des livres gratuits, dont «l'acte de libération » est « au cœur» de la pratique. Et d'ajouter : « Notre but, tout simplement, est de faire du monde une bibliothèque! Le bookcrossing est un moyen d'échange des livres aux proportions infinies ». On reconnaît déjà dans ces quelques phrases tous les éléments de l'idéologie véhiculée par Internet (l'ubiquité et l'abolition des limites spatio-temporelles) Le slogan du site français est: "Libérez les livres!" Les acteurs se définissent comme des «passeurs», des «chasseurs» mais aussi en français, des « bookcorsaires », des flibustiers du livre donc, des pirates de la lecture. Et en effet, l'action des « bookcorsaires » et leur vision du monde du bookcrossing sont guidées par un imaginaire qui tourne autour de la récente philosophie du "libre » qu'il faut comprendre à travers les deux traductions anglo-saxonnes du même mot (free) : tout est gratuit et chacun est libre de participer.

\footnotetext{
${ }^{1}$ Le site $W e b$ accueille une rubrique « shopping » où les internautes peuvent acheter tout le matériel nécessaire à l'activité du bookcrossing (étiquettes, affiches, etc.). Ils peuvent aussi cependant les créer eux-mêmes et les partager sur le «Wiki des bookcrosseurs», ce qui est le cas dans le bookcrossing français. Des liens commerciaux sont aussi proposés vers des librairies en ligne.

${ }^{2}$ Voici quelques exemples de messages que l'on peut trouver : «Emportez-moi ! »; « je ne suis pas perdu »; « ce livre a été laissé ici pour trouver un nouveau lecteur »,...

${ }^{3}$ Voir Wikipedia, encyclopédie en ligne dite «collaborative » dont la fiabilité est controversée car elle se définit comme «Projet d'encyclopédie librement réutilisable que chacun peut améliorer» (http://fr.wikipedia.org, consulté le 13 avril 2008). Voir aussi toute la vague actuelle des « logiciels libres » dont la particularité est qu'ils sont libres de droit et libres d'accès.
} 
Ne pourrait-on pas voir dans cette nouvelle pratique de la culture urbaine, une illustration du mouvement dialectique de délocalisation/relocalisation propre à la société urbaine contemporaine ? Dans les sociétés «pré-modernes » telles que les a décrites Anthony Giddens, le local primait le global : l'intimité, les affects se vivaient dans l'ici et maintenant. À l'inverse, dans les sociétés hypermodernes, le développement des médiations techniques a entraîné la fragmentation, la délocalisation des individus, des liens sociaux mais aussi simultanément leur relocalisation (Internet atteste la distance tout en l'abolissant). Comme l'a montré Anthony Giddens, on confie à des systèmes experts le soin de gérer la vie courante. Cependant, cette dépossession au profit des spécialistes s'accompagne dialectiquement d'une réappropriation au niveau des individus. Ainsi Internet qui représente un système expert par excellence sert simultanément à délocaliser et à re-localiser l'activité.

Dans le cas du bookcrossing, précisément, on trouve ce double mouvement dans lequel le distant peut se re-localiser dans la sphère de l'intime et du personnel et inversement le personnel, l'intime peut se distribuer, se téléporter au niveau du public. Cette dialectique agit sur plusieurs plans. Premièrement, la conséquence la plus visible de la conjonction d'Internet à la pratique du bookcrossing est la délocalisation de la bibliothèque traditionnelle et sa dématérialisation. A la localisation physique d'un ouvrage au sein d'un centre de documentation (ou d'une librairie), le bookcrossing oppose la singularité d'un livre "atopique », délocalisé et de surcroît non intégré dans une collection raisonnée. Le bookcrossing manifeste ainsi une volonté de déplacer la centralité des lieux où l'on peut habituellement trouver un livre.

Deuxièmement, la technologie Internet est omniprésente pour gérer le traçage des livres, organiser les manifestations comme les «méga bookcrossing» (grande chasse au livre), les "bancs à livres » ou encore les " arbres à livres », enfin pour communiquer ses goûts littéraires. Le système des relations humaines est d'abord distant et anonyme puisque relayé (pseudos, forums). Des rencontres ont lieu entre les bookcrossers mais elles n'arrivent que dans un second temps et de manière organisée (heure et lieu de rendez-vous fixés collectivement sur les forums ou "pm», c'est-à-dire les "private messages " (courriel indirect). Ainsi, en dédoublant les actions des bookcrossers, en les consignant sur le site Web de l'activité, Internet produit comme une seconde "vie» en décalé, discontinue, un peu fantomale, à la fois spectaculaire et masquée.

En retour, il y a une image de la cité reconstruite à travers le découpage des villes en des zones urbaines où sont déposés les livres. Ces dernières qui représentent des points de rendez-vous réels deviennent les pièces d'un puzzle imaginaire et infini transposé sur la toile. Par le réseau Internet, des morceaux de ville sont comme cousus ensemble pour former une mosaïque urbaine mondiale (Jardin de la Fontaine à Nîmes-Gare de Lyon à Paris-Polygone à Montpellier-Kensington Park à Londres, etc.). Ainsi a lieu une relocalisation imaginaire dans une cité patchwork où les distances sont abolies; image d'une société-ville réticulaire ${ }^{1}$, une cité globale du livre multilingue offert, échangé, trouvé.

Se dessine alors un idéal de société, une u-topie: une communauté des échanges libres, de l'entente parfaite et de l'amitié interplanétaire au cœur de laquelle la ville devient objet affectif et esthétique (le mobilier et le décor urbain — banc, arbres, cages d'escalier,... — deviennent à ce propos des lieux d'exposition « sauvages » pour le livre). La ville apparaît comme creuset de la création, de l'imagination, comme lieu de culture et des possibles. C'est la ville des lieux publics, la ville hédoniste, la ville des flâneurs ${ }^{2}$, la ville des rencontres

\footnotetext{
1 Cf. André Akoun, «Nouvelles techniques de communication et nouveaux liens sociaux», Cahiers Internationaux de Sociologie, op. cit., p. 11.

2 «Une ivresse s'empare de celui qui a marché longtemps sans but dans les rues. » (Walter Benjamin, Paris, capitale du XIXe siècle : le livre des passages, trad. de l'allemand par Jean Lacoste, Paris, éd. du Cerf, 1997, p. 434). Cette phrase est extraite du chapitre sur « le flâneur » (ibid., 434-472).
} 
fortuites, la ville surréaliste des trouvailles, la ville de l'imprévu et de la nouveauté. C'est aussi la ville produit de la civilisation naturalisée qui devient « nature urbaine », comme l'ont bien montré les travaux en écologie urbaine ${ }^{1}$. Ce n'est probablement pas un hasard si les bookcrossers parlent de livres «lâchés dans la nature » ou "released in the wild», selon la formule originale en anglais. Donc, une ville milieu-naturel, une ville sauvage.

Dans cette recomposition territoriale rêvée, n'y a-t-il pas aussi la recherche d'une relocalisation symbolique du soi, selon une contemporanéité désirée entre le lecteur-passeur et le lecteur-receveur? Le livre voyageur, sans localisation fixe, apparait comme point de contact physique, comme lieu tactile entre des protagonistes distants. Objet-relais, il devient alors à lui seul une sorte de territoire de la rencontre. Idéalement, il devrait s'agir du livre que j'ai aimé et dont je me déleste au profit d'un lecteur inconnu' ${ }^{2}$. Ce lecteur représente une chance de déplacement et d'aventure. Le mouvement de cette circulation implique ainsi un livre, autrui et moi dans la ville.

Or, il semble que l'on puisse voir s'exprimer là quelque chose de l'ordre d'une singularisation $^{3}$, d'une originalité du soi qui rappelle l'autre dimension de l'individualisme moderne vue par Georg Simmel. Le sujet moderne nécessairement urbain connaît deux faces : si la modernité a produit "l'indépendance individuelle» (qui œuvre dans le sens de la distanciation généralisée), elle provoque aussi le désir de «l'originalité personnelle ${ }^{4}$ » (recentrage). Il est d'ailleurs significatif que les bookcrossers assidus aient leurs propres «blogs », minis sites «perso » où chacun se met en scène, se raconte, se fabrique un égomonde (" mes lectures du mois », " ce que j'ai aimé », " ce que je déteste », " mes photos », « mes liens », etc.) Le blog comme nouvelle présentation de soi $^{5}$ et comme affirmation de « « L'unicité qualitative et le caractère irremplaçable ».

\section{Face aux TIC : la piste de la singularisation}

Cette double singularité affirmée - ce que Georg Simmel a défini comme «L'unicité qualitative et le caractère irremplaçable ${ }^{6} »$ - à la fois du livre choisi et du lecteur qui s'exprime, évoque une re-localisation symbolique et affective: quelque part dans un environnement urbain, il y a un livre qui $m$ 'attend. En même temps qu'Internet représente une mise à distance, il entre dans mon intimité, dans ma vie privée. C'est chez moi que j'éprouve le plaisir de la nouveauté, l'exaltation de la promesse d'une rencontre avec un objet passé par d'autres mains, aimé par une autre âme anonyme, un passant sorti de la foule pour déposer ce cadeau qui me désigne comme l'élu. Ainsi cette personnalisation du rapport à un livre - qui

\footnotetext{
${ }^{1}$ Voir Yves Grafmeyer et Isaac Joseph (sous la dir. de), L'école de Chicago : naissance de l'écologie urbaine, Paris, Flammarion, 2004, pp.9-11. Voir aussi Thierry Paquot pour qui : «La nature de notre culture est dorénavant urbaine » (Vive la ville, Condé-sur-Noireau, Arléa-Corlet, 1994, p. 12.)

${ }^{2}$ En réalité, on s'aperçoit à fréquenter les bookcrossers que le désir « d'être trouvé » étant plus le plus fort, on s'autorise à passer outre cet idéal... Du coup, les livres qui sont « relâchés » n'ont parfois jamais été lus. Il arrive fréquemment que des membres achètent des ouvrages au rabais pour pouvoir les semer en quantité. Mais comme la réalité n'est jamais simple, ce sont aussi ces mêmes personnes qui lisent véritablement et se délestent de lectures appréciées.

${ }^{3}$ Sur «l'hypothèse de la singularité », voir Patrick Baudry, Violences invisibles: corps, monde urbain, singularité, op. cit., p. 165 et suiv.

${ }^{4}$ Georg Simmel, « Métropoles et mentalité » (1903), in Yves Grafmeyer et Isaac Joseph (sous la dir. de), op. cit., p. 63 .

5 Pour une analyse détaillée des « pages perso » et des blogs comme expression du soi, voir : Laurence Allard et Frédéric Vandenberghe, «Express yourself! Les pages perso: entre légitimation technopolitique de l'individualisme expressif et authenticité réflexive peer to peer», Réseaux, 2003/1, n 117, pp. 191-219; Dominique Cardon et Hélène Delaunay-Teterel, « La production de soi comme technique relationnelle : un essai de typologie des blogs par leurs publics », Réseaux 2006/4, n 138, pp. 15-71.

${ }^{6}$ Georg Simmel, op. cit., p. 63.
} 
n'est pas nouvelle et s'est toujours pratiquée de manière informelle dans l'échange amical quotidien ${ }^{1}$-, s'érige en principe de vie et à grande échelle.

C'est à ce niveau que l'imaginaire joue à plein. Il y aurait de quoi se demander si dans cette démarche, il ne faudrait pas voir une forme de résistance douce de l'individu contre un certain nombre de traits des sociétés modernes communément désignés comme négatifs : la planification à quoi répondrait l'imprévu, la rationalisation à quoi répondrait la subjectivité du goût personnel, la capitalisation à quoi répondrait le délestage, la massification à quoi enfin répondrait l'expression personnelle. En même temps, pour le lecteur internaute, il y a aussi la clandestinité, l'anonymat en réponse au fichage, à l'identification administrative, etc. Le bookcrossing trouve en effet sa place comme réappréciation d'un espace, je dirais même de deux formes d'espace entre lesquels il y a un lien : l'espace public de la ville qui est une expression de l'espace social en général, et l'espace intime privé du chez soi, socialement perçu comme un espace psychologique. Tout se passe comme si l'individu avait compris que la société que nous vivons a étendu son pouvoir jusque dans nos sphères intimes (autrement dit, le dedans) et qu'en réponse à cela, il avait fallu qu'à son tour il investisse l'espace social dont l'expression la plus immédiate est le lieu urbain (le dehors), pour récupérer un peu de ce qui lui aurait été enlevé. La ville qui accueille le double corporel du passeur, c'est-à-dire le livre qui lui a appartenu, qu'il a lu et manipulé, apparaît comme le lieu de récupération d'une forme d'intimité et de propriété non plus matérielle mais psychique, quelque chose de l'ordre de l'ipséité.

Dans ce mouvement dialectique, le décentrement géographique (du lieu d'achat ou de consultation du livre) est remplacé par une relocalisation symbolique : elle instaure le territoire du livre fondé non plus sur une rationalité monétaire ou pratique (emprunter au plus près) mais sur des considérations affectives et idéalistes : l'amour de la lecture, l'imaginaire de la rencontre, le bonheur de la trouvaille (un peu comme les œufs surprise cachés dans le jardin à Pâques), la volonté du non choix et la recherche de l'aventure, du risque (certes faible), le plaisir de l'abandon au hasard, à l'irrationnel et à l'inconnu — tout cela plus que la possession d'un contenu ou l'acquisition d'une ne référence précise qu'on irait chercher dans une bibliothèque ou une librairie. C'est même l'expérience exaltante de la dépossession (encore qu'il n'est pas sûr en réalité qu'on se défasse toujours d'un livre auquel on tient...) dans le don, l'offrande personnelle à un anonyme, mais aussi l'attente.

L'internaute qui visite le site du bookcrossing ainsi que les blogs des fervents adeptes s'apercevra bien vite que l'excitation du jeu se situe autant dans le dépôt, la cession que dans la trouvaille. Faut-il comprendre ce vécu comme une manifestation, voire une revendication symbolique du soi, de sa corporéité et d'une " singularité quelconque ${ }^{2}$ dans l'espace de la modernité urbaine, à travers un acte signifiant: le don d'un objet fétiche, le livre qu'on a aimé, autrement dit une lecture qu'on a incorporée, qui exprime une part de soi et qu'on offre au " premier venu »? Le vocabulaire chargé de sens affectif utilisé par les adeptes est assez révélateur : on parle d' « abandonner », de « libérer», de « lâcher» ou de " chasser » un livre dont on voudrait après suivre la « vie »; comme s'il s'agissait de rendre la liberté à l'ouvrage prisonnier d'une étagère, d'une maison, d'une collection. L'anthropomorphisme qu'on prête à l'objet-livre renvoie à son incorporation. Abandonner un livre " dans la nature », comme il est dit sur le site, n'est-ce pas un peu comme s'abandonner soi-même entre les mains du hasard, s'abandonner aussi au déplacement, au transport vers un ailleurs encore inconnu ? Ce rêve non dit d'abolir le sens de la propriété, à travers la circulation gratuite d'un livre, passant entre

\footnotetext{
${ }^{1}$ Christophe Evans, en 1992, traitait déjà dans un article de « La circulation privée des livres entre les lecteurs : joindre l'utile au convivial », Bulletin des Bibliothèques de France (BBF), tome 37, n 1, 1992, pp. 26-31.

${ }_{2}$ Giorgio Agamben, La communauté qui vient: théorie de la singularité quelconque, trad. de l'italien par Marilène Raiola, Paris éd. du Seuil, 1990, p. 9.
} 
les mains d'un «cercle invisible de lecteurs » ${ }^{1}$ évoque à la fois un idéal du prochain (le premier venu qui n'est pas mon intime) et une érotique de l'abandon: pas d'attaches, pas de frontières, pas de distances et le choix de laisser le choix à « la fée Occasion $»^{2}$.

La modernité urbaine, qui ne désigne pas que des avancées technologiques mais aussi la formation d'un mode de conscience, s'est radicalisée avec l'apparition et l'usage d'Internet. Georg Simmel, il y a cent ans, avait déjà repéré les structures de la mentalité métropolitaine qui valent encore aujourd'hui : l'esprit rationnel propice aux abstractions (économique, intellectuelle,...) mais aussi sa contradiction dans l'affirmation de l'âme romantique. L'introduction et la démultiplication des images dans le monde social participent de ce mouvement. Elles ont amené une évolution dans les formes de socialisation, étendant encore le pouvoir de l'urbanité, pénétrant nos corps et faisant de nous des sujets sociaux imagés et pensant en images.

A côté, voire en réaction aux rationalisations numériques (identifications, fichages, répertoires,...) imposées par une société gestionnaire et technocrate, en réponse à l'abstraction de soi dans la vie active, attitude de l'homme blasé simmelien, naissent en marge des formes de sociabilité à visée alternative. Les modes du rencontrer qui touchent à la sphère privée n'y échappent pas. Des tentatives de relocalisation existent ainsi avec plus ou moins de succès et plus ou moins d'autonomie par rapport aux systèmes experts qui gèrent à distance. Ainsi les speed dating amoureux ou en vue d'un recrutement visent-ils à redonner un sens local et physique à la rencontre. Mais en tant que système à rendement, ils reproduisent le pendant rationnel de la modernité. Le jeu du local et du global, de la proximité et de la distance est plus complexe dans le cas du bookcrossing, pratique culturelle autour de la lecture, se déroulant au cœur de ce que nous pourrions appeler " l'urbanité numérique ${ }^{3} »$. Le désir de singularisation qui s'y exprime suggère la recherche d'une relocalisation symbolique du sujet à soi. Dans cette activité, la rencontre occupe une place à la fois de premier plan et d'arrière-plan : elle n'est pas tant une action voulue entre les participants qu'une « ambiance » qui accompagne la pratique. Une atmosphère de rencontre, avec ses effets de hasard, d'aventure, de coïncidence, entoure l'expérience du bookcrossing.

Curieusement, derrière ces tentatives de réapproriation subjective, derrière le retour à une proximité rêvée et reconstruite (par le biais du Web), apparaît une sorte de vide au niveau des relations interpersonnelles. Il reste en effet le principe de la téléprésence structurant les contacts, ce qui pose question. Pour Paul Virilio, elle constitue « une menace considérable de perte de l'autre $»{ }^{4}$. C'est pourquoi, derrière la séduction du passe-livre, on se demande dans quelle mesure le livre qui voyage, qui passe indirectement de main en main ne représenterait pas aussi une manière de reporter la rencontre effective d'autrui, toujours plus loin, toujours plus tard...

Cécile Duteille

Docteur en Sociologie -

GERPHAU (Philosophie, Architecture, Urbain) - Ecole d'Architecture de Clermont-Ferrand.

\footnotetext{
${ }^{1}$ C'est le slogan du Passe-livre, l'autre site d'échanges dont j'ai parlé plus haut.

${ }^{2}$ Vladimir Jankélévitch et Béatrice Berlowitz, Quelque part dans l'inachevé, Paris, Gallimard, 1990, p. 41.

${ }^{3}$ Dominique Boullier, L'urbanité numérique : essai sur la troisième ville en 2100, Paris, L'Harmattan, 1999.

${ }^{4}$ Paul Virilio, Cybermonde : la politique du pire, entretien avec Philippe Petit, Paris, Textuel, 2001, p. 45.
} 